\title{
Linear Stability Analysis of Mixed Convection Under Soret Effect in Bounded Horizontal Fluid Layer with Slip Conditions
}

\author{
A. Kadiri, H. Ben Hamed, H. Beji \\ LTI Laboratory, Jules Verne University of Picardie, Amiens, France
}

Email address:

anass.kadiri@u-picardie.fr (A. Kadiri), haykel.benhamed@u-picardie.fr (H. B. Hamed), hassen.beji@u-picardie.fr (H. Beji)

To cite this article:

A. Kadiri, H. Ben Hamed, H. Beji. Linear Stability Analysis of Mixed Convection Under Soret Effect in Bounded Horizontal Fluid Layer with Slip Conditions. American Journal of Mechanics and Applications. Vol. 3, No. 3, 2015, pp. 19-26. doi: 10.11648/j.ajma.20150303.11

\begin{abstract}
The aim of this study is to analyze the mixed convection under Soret effect in a liquid layer subjected to adverse temperature and concentration gradients and slip conditions. Theoretical linear stability analysis and numerical methods were performed to study the convective and absolute instabilities of the transverse rolls. It is found that, for convective instabilities, the problem has analytical solution and is a Galilean transformation of natural convection. The throughflow makes the rolls to propagate and the frequency shift is proportional to Reynolds number. For higher Rayleigh numbers, advection breaks Galilean invariance, and absolute instabilities settle. At the threshold of absolute instability, the presence of throughflow breaks the symmetry by the rotational invariance, and the system selects among an infinite number of unstable modes, the progressive waves, in the direction of the flow.
\end{abstract}

Keywords: Mixed Convection, Horizontal Liquid Layer, Critical Rayleigh, Linear Stability Analysis

\section{Introduction}

Stability of mixed convection of multicomponent fluids with two free boundaries is very important in numerous physical phenomena. It spans diverse fields such as heavy pollutants discharged into watercourses and generating exothermic reactions, storage of solar energy in salty pools, natural environments with stratified salinity gradient in lakes, and some daily world experiments such as Gulf Stream or Gibraltar currents or evaporation and transport in salt lakes See Nield and al. (2008) [1]. Indeed, the Mediterranean Sea evaporates and grows saltier, cold seawater coming from the Atlantic crosses the Strait and flows over the warmer salty water. Vertical temperature and concentration gradients act together in presence of the forced Atlantic flow sliding in motion with free-free conditions. At the idealized situation, the problem is analog to Poiseuille-Rayleigh-Bénard-Soret (PRBS) Ben Hadid, and Henry (2007) [2], but it is quite different due to the absence of hydrodynamic boundary layers. Despite the difficulties of realizability of laboratory quantitative experiments even under very artificial conditions, this case is theoretically interesting because it allows an explicit and clearly solution.

A detailed linear stability analysis of natural convection in multicomponent systems is found in the book of Platten and Legros (1984) [3]. When the separation ratio $\mathrm{N}$, characterizing the Soret effect, is greater than a critical value $N^{\star}$, convection is stationary. However, when $\mathrm{N}$ is lower than $N^{\star}$ the system crosses a Hopf bifurcation.

The superposition of the average flow with the vertical gradient of temperature changes the nature of convection mode. Besides convective instabilities, absolute instabilities can take seat. A convective instability amplifies any unstable disturbance, and advects it towards the downstream; regarding the disturbances as a noise. A convective unstable system behaves like a noise amplifier. On the other hand, an absolute unstable system answers a forcing selectively. Its answer is dominated by the mode of vanished speed of group; the other modes are swept by the flow. The dominating mode being selected by the dispersion relation, which is intrinsic to the system. An absolute unstable system behaves like an oscillator with its Eigen frequency.

Müller et al. (1992) [4] were just regarded the free-free case as a Galilean transformation leading to a simple frequency shift. Ben Hamed et al. (2009) [5] showed via a linear stability analysis and direct numerical simulations in a semi-infinite cavity, that the length of establishment of thermal entry zone in the case of free-free boundaries varies 
almost linearly with the Peclet number, and is significantly lower than the case of rigid boundaries.

Carrière and Monkewitz (1999) [6] have studied convective versus absolute instabilities in PRB configuration. Their main result is that the mode reaching zero group velocity at the convective-absolute transition always corresponds to transverse rolls, while the system remains convectively unstable with respect to pure streamwise (longitudinal) rolls for all non-zero Reynolds numbers. We mention in passing that this result is similar to which obtained in free-free case.

Joulin and Ouarzazi (2000) [7] studied the absolute instabilities in the case of mixed convection of a binary mixture saturating a Darcy medium. This case is very similar to the present problem due to the absence of the boundary layer. They showed that in the presence of the filtrating flow, the transverse rolls is the exclusively selected mode, and that progressive waves going back the througflow have the lowest absolute threshold.

In practice, one way to ensure the slip conditions is to consider immiscible liquids arranged in layers, the studied liquid is floating on top of a somewhat denser liquid. In general the authors take into account surface tension, which does not fit to the scope of this work. Nevertheless, Campbell and Koster (1995) [8], studied two fluid layers, and have numerically shown, using a finite element method, that the presence of the upper layer reduces strongly the convection in the lower one, which limits the deformations on interface between the two liquids. The results of the results of Campbell and Koster (1995) [8] are very interesting to imagine a laboratory experiment with free-free conditions.

In the present work, we make stability analysis of mixed convection in semi-infinite layer of a binary mixture. We study the convection without restriction of direction of propagation. The objective is to find an explicit analytical expression of critical Rayleigh as function of all the physical parameters of the fluid and the flow. It is either found, that due to advection, the transport mechanism cannot be considered as a simple frequency shift. Forced flow induces an area of convective instability and another area of absolute instability when buoyancy ratio $\mathrm{N}$ is lower than Nc. Otherwise, there is a critical Peclet, which depends on $\mathrm{N}$, beyond which the Soret effect is suppressed. The selected mode is corresponding to the transverse rolls. The study of the spatial branches shows that progressive waves propagating in the direction, and against the throughflow, are equally probable.

\section{System and Hypothesis}

The considered geometry is a semi-infinite horizontal layer of thickness $L_{z}$ containing a binary Boussinesq incompressible liquid (Fig.1) with free upper surface and sliding on a heavier liquid. The liquids are immiscible, and surfaces are undeformable. A steady adverse temperature and concentration gradients are maintained, and a lateral pumping drives the flow uniformly on the $x$ direction. Soret effect is considered and the flow is assumed to be laminar. The table 1 summarizes the main scaled variables of the system.

$$
u_{C}=P e \longrightarrow T=C=0, \psi=P e, \quad \frac{\partial^{2} \psi}{\partial z^{2}}=0
$$

Figure 1. The system at the basic conductive state.

Table 1. Reference values.

\begin{tabular}{llll}
\hline Physical entity & Reference value & Dimension & Non dimensional value \\
\hline Density & $\rho_{\text {ref }}$ & {$\left[\mathrm{kg} \cdot \mathrm{m}^{-3}\right]$} & $\frac{(x, z)}{L_{z}}$ \\
Distance & $L_{z}$ & {$[\mathrm{~m}]$} & $\frac{(u, w) \times L_{z}}{\kappa}$ \\
Velocity & $V_{\text {ref }}=\frac{\kappa}{L_{z}}$ & {$\left[\mathrm{~m} \cdot \mathrm{s}^{-1}\right]$} & $\frac{t^{\star} \times d}{L_{z}^{2}}$ \\
Time & $t_{\text {ref }}=\frac{L_{z}^{2}}{\kappa}$ & {$[\mathrm{s}]$} & $\frac{2 \times T^{\star}}{\left(T_{\text {hot }}-T_{\text {cold }}\right)}$ \\
Temperature & $T_{\text {ref }}=\frac{1}{2}\left(T_{\text {hot }}-T_{\text {cold }}\right)$ & {$[K]$} & $\frac{C-C_{\text {ref }}}{C_{\text {ref }}}$ \\
\hline
\end{tabular}


The dimensional form of equations is written:

$$
\left\{\begin{array}{c}
\vec{\nabla} \cdot \vec{V}=0 \\
\partial_{t} \vec{V}+(\vec{V} \cdot \vec{\nabla}) \vec{V}=-\nabla P+\operatorname{Pr} \nabla^{2} \vec{V}+\operatorname{Ra} \cdot \operatorname{Pr}(T+N C) \cdot \vec{k} \\
\partial_{t} T+(\vec{V} \cdot \vec{\nabla}) T=\nabla^{2} T \\
\partial_{t} C+(\vec{V} \cdot \vec{\nabla}) C=\frac{1}{L e}\left(\nabla^{2} C-\nabla^{2} T\right)
\end{array}\right.
$$

The problem is controlled by five parameters namely: Rayleigh number $\Re=t_{\text {stab }}^{2} / t_{\text {destab }}^{2}$, where $t_{\text {stab }}=H^{2} / \sqrt{\kappa \nu}$ is the stabilization time, due to the mechanisms of evacuation and dissipation of energy, and the destabilization time $t_{\text {destab }}=\sqrt{H / \beta \Delta T g}$ due to Archimedes' effect. Reynolds number $R e=\frac{u L_{Z}}{v}$ which relates inertial forces to viscous ones, Buoyancy ratio $N=S_{T} \times\left(\frac{\beta_{\phi}}{\beta_{T}}\right) C_{0}\left(1-C_{0}\right)$ which quantifies separation of species, Prandtl number $\operatorname{Pr}=\frac{v}{\kappa}$ and Lewis number $L e=\kappa / D_{C}$ which are material parameters of the liquid. The basic conductive state obey to a linear profile of temperature and concentration (eq. (2)), and constant velocity (eq. (3)).

$$
\begin{gathered}
T_{C}=C_{C}=1-z \\
u_{C}=\frac{U_{i n}^{\star}}{V_{r e f}}=\frac{U_{i n}^{\star} \times L_{z}}{\kappa}=\frac{U_{i n}^{\star} \times L_{z}}{v} \times \frac{v}{\kappa}=R e \times P r=P e
\end{gathered}
$$

\section{Eigenvalue Equation of Mixed Convection in a Two-Component Fluid}

The solutions of the system can be decomposed into a sum of the pure conductive and the convective ones. These forms are injected in the general equations in order to have the perturbation equations. In the foregoing analysis, we take the curl of momentum equation and differentiate again with respect to $x$, we obtains the linked Navier-Stokes, energy, and species equations written respectively in the small deviations $w, \theta$ and $\phi$ of vertical velocity, temperature and mass fraction.

$$
\left(\begin{array}{ccc}
-\nabla^{2}\left(\partial_{t}+P e \partial_{x}\right)+\operatorname{Pr} \nabla^{4} & \operatorname{RaPr}\left(\partial_{x}^{2}+\partial_{y}^{2}\right) & N \operatorname{RaPr}\left(\partial_{x}^{2}+\partial_{y}^{2}\right) \\
-1 & \partial_{t}+P e \partial_{x}-\nabla^{2} & 0 \\
-1 & \frac{1}{L e} \nabla^{2} & \partial_{t}+P e \partial_{x}-\frac{1}{L e} \nabla^{2}
\end{array}\right)\left(\begin{array}{c}
w \\
\theta \\
\phi
\end{array}\right)=-\left(\begin{array}{c}
\partial_{x}\left[w \nabla^{2} u-u \nabla^{2} w\right] \\
{\left[u \partial_{x}+w \partial_{z}\right] \theta} \\
{\left[u \partial_{x}+w \partial_{z}\right] \phi}
\end{array}\right)
$$

The left-hand side operator is linear. The right-hand side terms are nonlinear. The perturbation dimensionless boundary conditions on horizontal borders are:

$$
\theta=\phi=\frac{\partial^{2} w}{\partial z^{2}}=w=0
$$

meaning respectively constant temperature and concentration, slip and non-deformability conditions. Linear analysis suggests that nonlinear terms are neglected, and then the right-hand side of system (4) is

$$
\begin{aligned}
& f=\hat{f} \sin (\pi z) e^{i\left(\Omega t+k_{x} x+k_{y} y\right)}+C C \\
& \mathcal{L}=\left(\begin{array}{cc}
a^{2}-i a \omega \operatorname{Pr}^{-1} & -\operatorname{Rak}^{2} \\
-1 & -a+i \omega \\
-1 & \frac{1}{L e} a
\end{array}\right.
\end{aligned}
$$

where $\mathcal{L}$ is the adjoint operator to the normal mode $f$. Finding the core of $\mathcal{L}$ leads to the complex dispersion

$$
\mathfrak{D}=a\left(a-i \omega \operatorname{Pr}^{-1}\right)(a-i \omega)+\operatorname{Rak}^{2}\left(1+N \frac{a(1+L e)-i L e \omega}{a-i L e \omega}\right)=0
$$

In the case of infinite Layer there is only established rolls, thus $\mathrm{k}$ is real. As will be shown in results and discussions $\omega$ is also real for all positive Rayleigh and $N$. In such situation the dispersion relation should be greatly simplified, and yields: 


$$
\begin{gathered}
\mathfrak{R} e(\mathfrak{D})=\frac{1}{(1+N(1+A))^{2}+(N B)^{2}}\left[\frac{\left(a^{3}+a \omega^{2}\right.}{\operatorname{Im}(\mathfrak{D})=\omega\left(\omega+\Omega^{+}\right)\left(\omega+\Omega^{-}\right)=0}\right. \\
\left\{\begin{array}{l}
A=\frac{L e a^{2}}{a^{2}+L e^{2} \omega^{2}} B=\frac{L e^{2} a \omega}{a^{2}+L e^{2} \omega^{2}} \omega=k_{x} P e+\Omega \\
\Omega^{ \pm}= \pm \sqrt{\frac{\left(\left(1+P r^{-1}\right)[1+N(1+L e)]+N L e^{2}\right) a^{2}}{L e^{2}\left(1+N+P r^{-1}\right)}}
\end{array}\right.
\end{gathered}
$$

In absence of flow, a trivial solution of $\operatorname{Im}(\mathfrak{D})=0$ is $\Omega=0$. The other two solutions, corresponding to a Hopf bifurcation, can exist only if $N<N^{\star}$ defined by

$$
N^{\star}=-\frac{1+P r}{(1+\operatorname{LePr})(L e+1)+\operatorname{Pr}}
$$

Stationary convection is then obtained for $N>N^{\star}$ and a wavenumber minimizing equation (9) gives the following critical Rayleigh

$$
R a_{C}=\frac{27 \pi^{4}}{4} \frac{1}{1+N(1+L e)}
$$

While the oscillating convection is obtained for $N<N^{\star}$, then, the system oscillates with the frequencies $\Omega^{ \pm}$and the oscillating Rayleigh is defined by:

$$
R a^{o s c}=\frac{27 \pi^{4}}{4} \frac{(1+L e P r)(L e+1) P r}{(L e P r)^{2}\left[1+L e\left(\frac{P r^{2}}{P r+1}\right)\right]}
$$

It is clear from the equation (10) that the spatial dependence of the system is not sensitive to the flow. The system undergoes a simple shift of frequency. However, at Rayleigh numbers higher than the critical one, the spatiotemporal evolution is different, and we can no longer consider the wave number and frequency as real. More, the through flow $P e$ generates two types of instabilities, namely, absolute and convective instabilities.

\section{Results and Discussion}

In the case of a pure fluid $N=0$, the equation (8) will be greatly simplified. Its minimization gives a unique solution shown in figure 2 . It corresponds to a steady state $(\omega=0)$ which critical threshold is the natural convection regardless of the flow Pe. The critical Rayleigh is $\mathcal{R}^{0}=\frac{27 \pi^{4}}{4}$ corresponding to a wave number $k=\frac{2 \pi}{\sqrt{2}}$. In natural convection the system has not a preferred direction. Any linear combination of the wave vectors $k_{x}$ and $k_{y}$ is a solution of the system See Chandrasekhar [9].

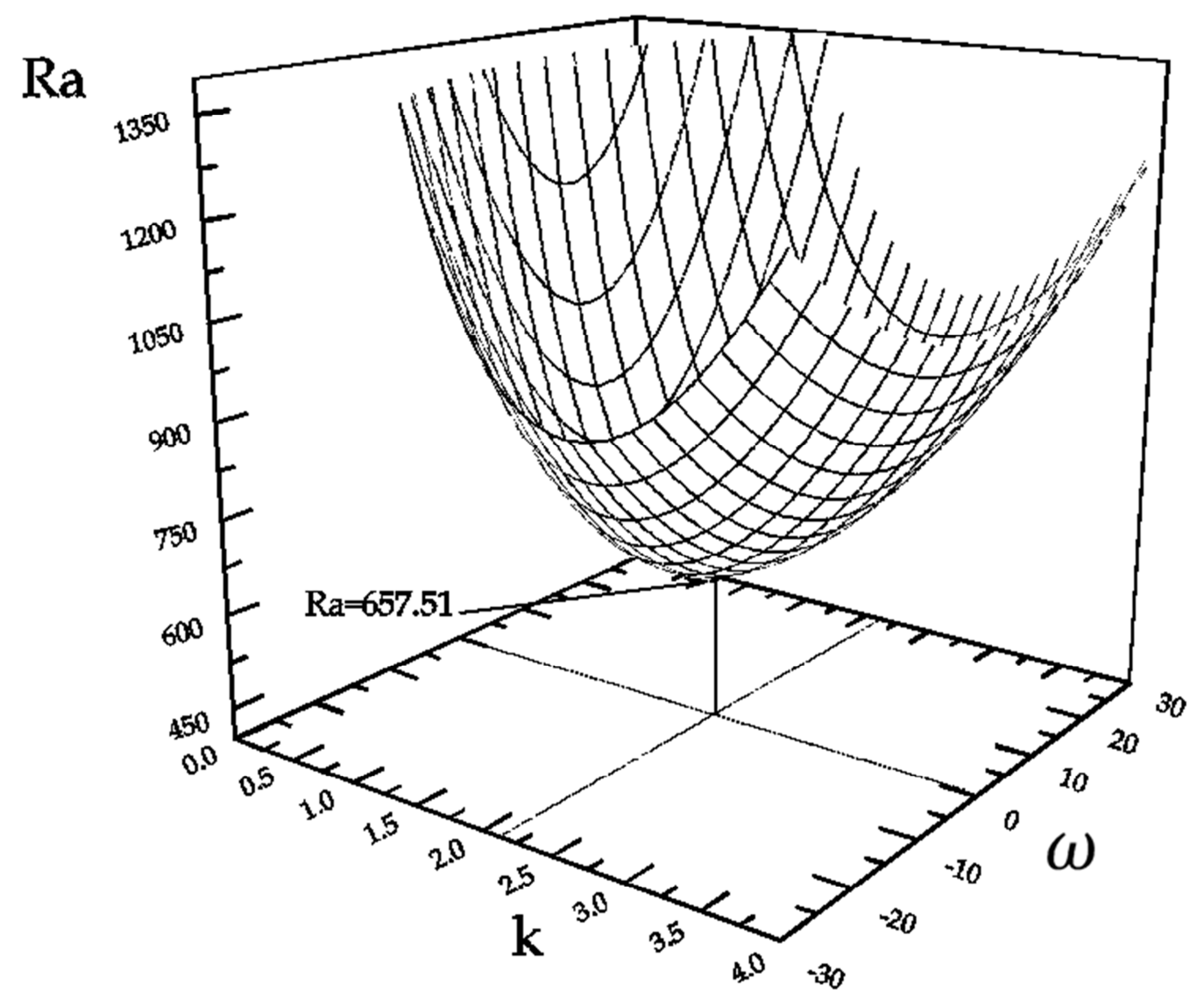

Figure 2. Neutral stability in the Ra-k- space: case of natural convection. 
When the through flow is applied on a binary mixture $N \neq$ 0 , a local disturbance tends to be evacuated if the group velocity $\frac{\partial \omega}{\partial k}$ is non-zero, this is called convective instabilities.

$$
\frac{\partial \omega}{\partial k}=0 \Rightarrow\left\{\begin{array} { c } 
{ \frac { \partial \omega } { \partial k _ { x } } = \frac { \partial \omega } { \partial k ^ { 2 } } \frac { \partial k ^ { 2 } } { \partial k _ { x } } - P e = 0 } \\
{ \frac { \partial \omega } { \partial k _ { y } } = \frac { \partial \omega } { \partial k ^ { 2 } } \frac { \partial k ^ { 2 } } { \partial k _ { y } } = 0 }
\end{array} \Rightarrow \left\{\begin{array} { c } 
{ 2 k _ { x } \frac { \partial \omega } { \partial k ^ { 2 } } - P e = 0 } \\
{ 2 k _ { y } \frac { \partial \omega } { \partial k ^ { 2 } } = 0 }
\end{array} \Rightarrow \left\{\begin{array}{l}
k_{x} \neq 0 \\
k_{y}=0
\end{array}\right.\right.\right.
$$

The system is then organised in transversal structures propagating with the pulsation $k_{x} P e+\Omega^{ \pm}$. Therefore, when the conduction state becomes absolutely unstable, progressive waves structured as transverse rolls are the only ones to be amplified locally. The other three-dimensional modes are amplified but convected downstream.

To determine the threshold of absolute instability, the dispersion equation (8) is solved with the assumptions:
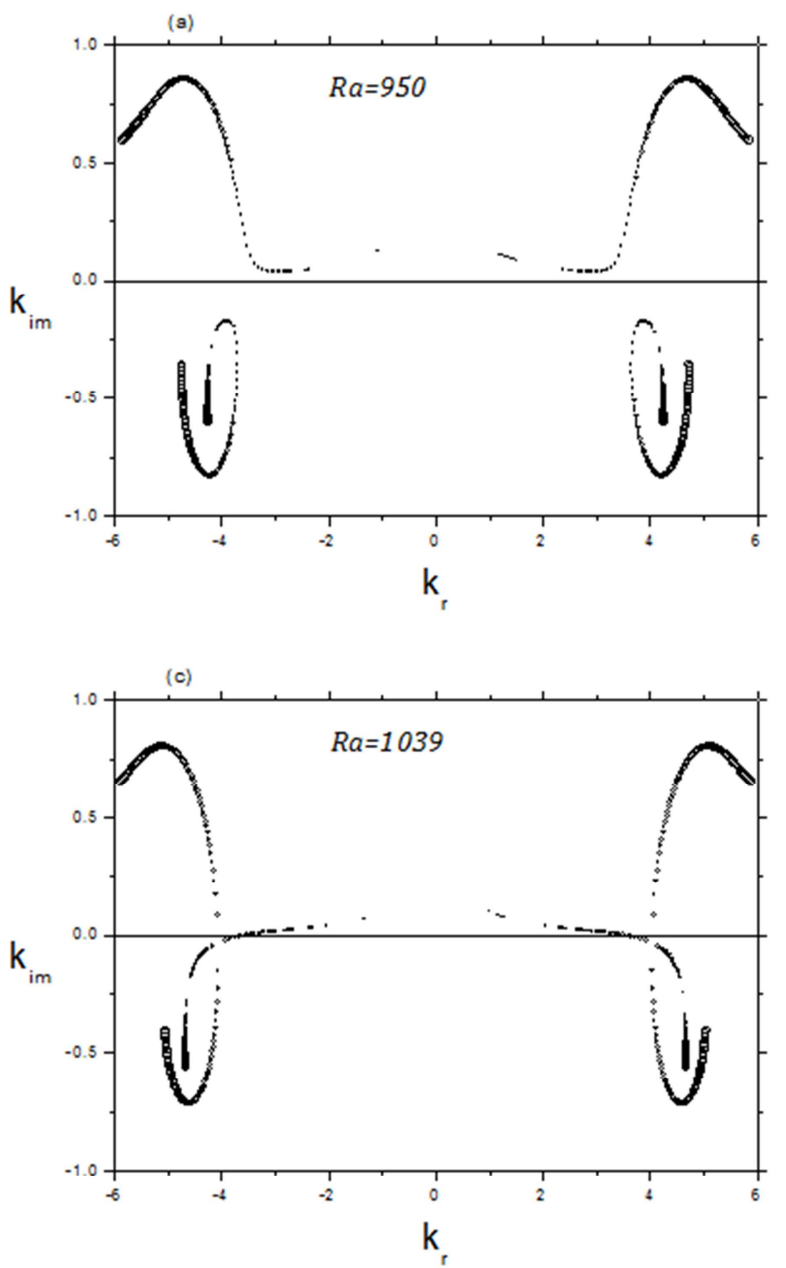

Local growth of instability requires vanished group velocity i.e.:

$k_{x} \in \mathbb{C}, \omega \in \mathbb{R}$ and $\mathcal{R}$ variable. The absolute Rayleigh number is reached at the pinching of spatial branches. The resolution is based on a numerical method of Newton Raphson. Figure 3 and 4 show clearly that the pinching of spatial branches is simultaneous for the $\omega^{-}$and $\omega^{+}$modes. In the absence of flow, a region of convective instabilities exists anyway.
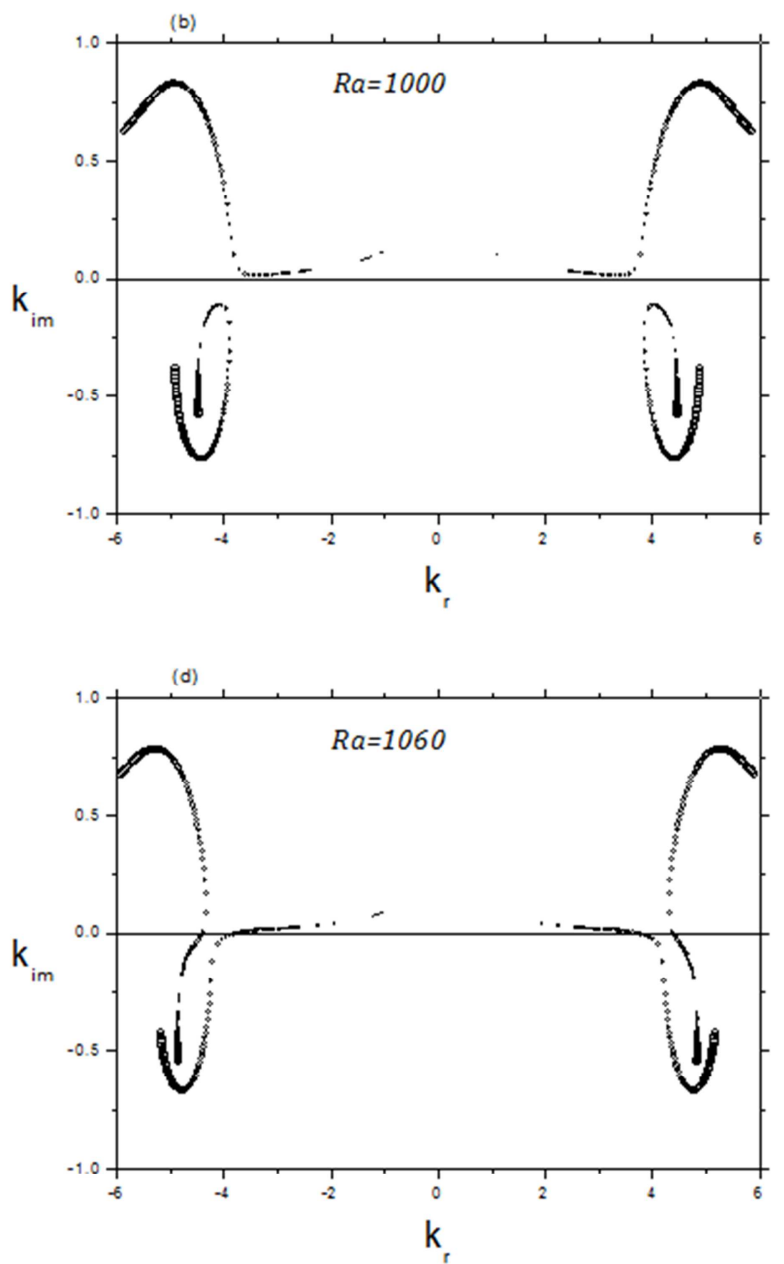

Figure 3. Pinching of the spatial branches of the dispersion relation in the plane $\left(k_{r}, k_{i}\right)$. Le $=140, N=-0.0006, \operatorname{Pr}=7, P e=1, R a_{a b s} \approx 1039(a) R a=$ 950, (b) $R a=1000,(c) R a=1039,(d) R a=1060$. 


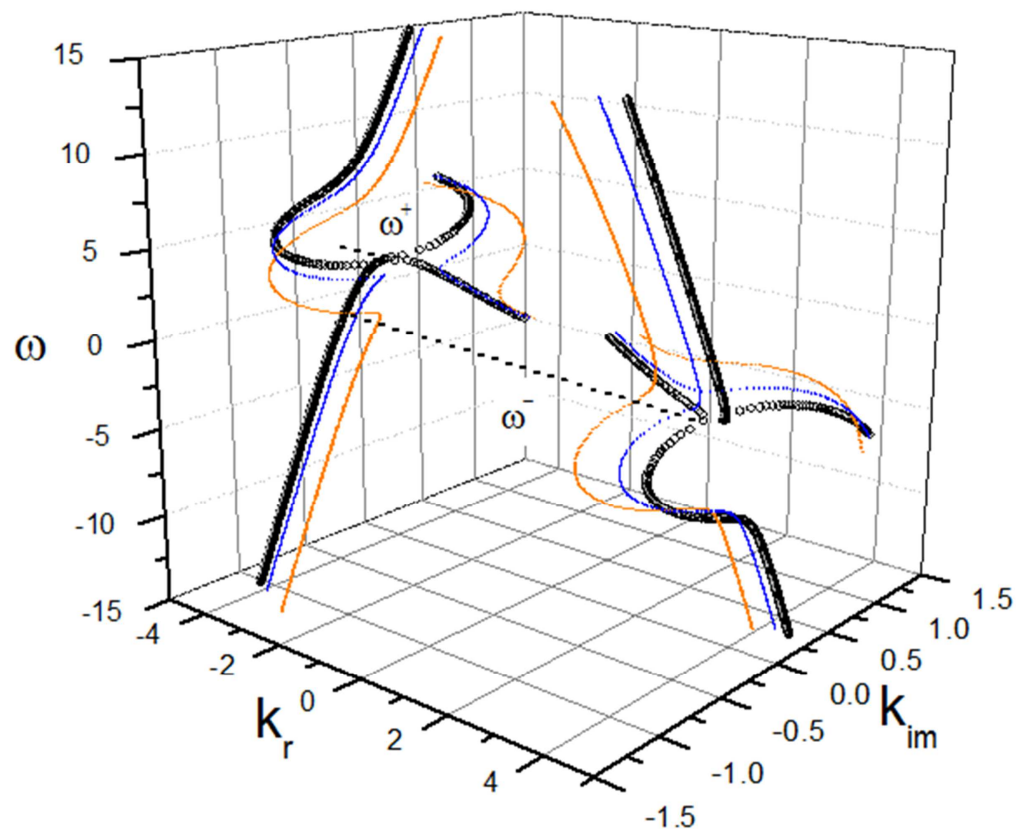

Figure 4. $3 D$ representation of the pinching of the spatial branches of the dispersion relation in the plane $\left(k_{r}, k_{i}\right) . L e=140, N=-0.0006, P r=7, P e=$ $1, R a_{a b s} \approx 1039$.

Figure 5 shows that for $\mathrm{N}>0$, there is a convective instabilities area bounded by supercritical threshold and a certain $P e^{\star}$ beyond which the Soret effect disappears. $P e^{\star}$ depends only on N. For negative separation rates, Figure 6 shows that the area of convective instability is more extensive. The absolute threshold is delayed with decreasing separation ratio. Figure 6 shows these absolute thresholds for different values of $N$. The two spatial branches involved in the pinching process for the cases $N=-0.0006, P e=$ $1, R a_{a b s}=1039$ were drawn in figure 3 .

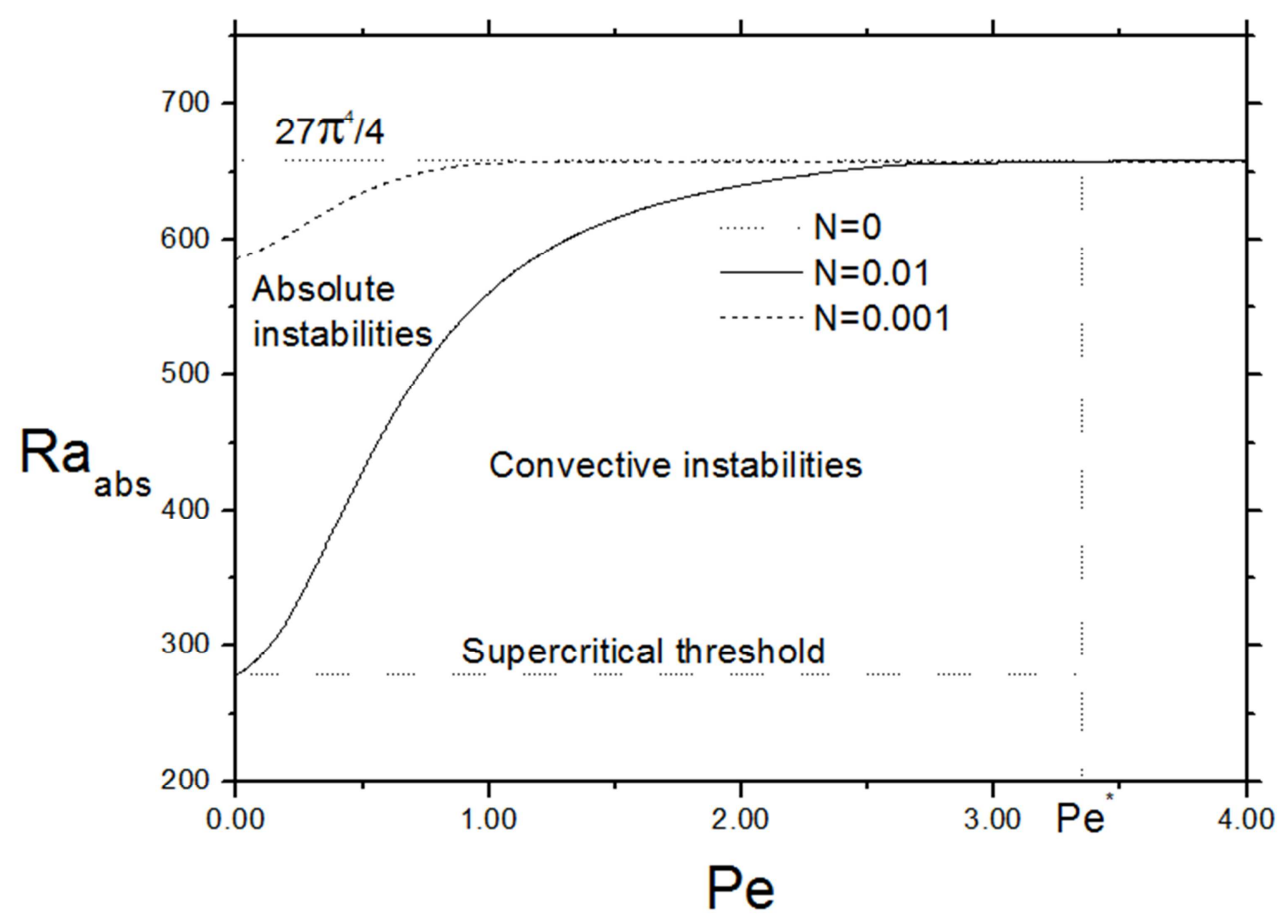

Figure 5. Variation of Ra with Pe for positive $N$, Le $=140$. 


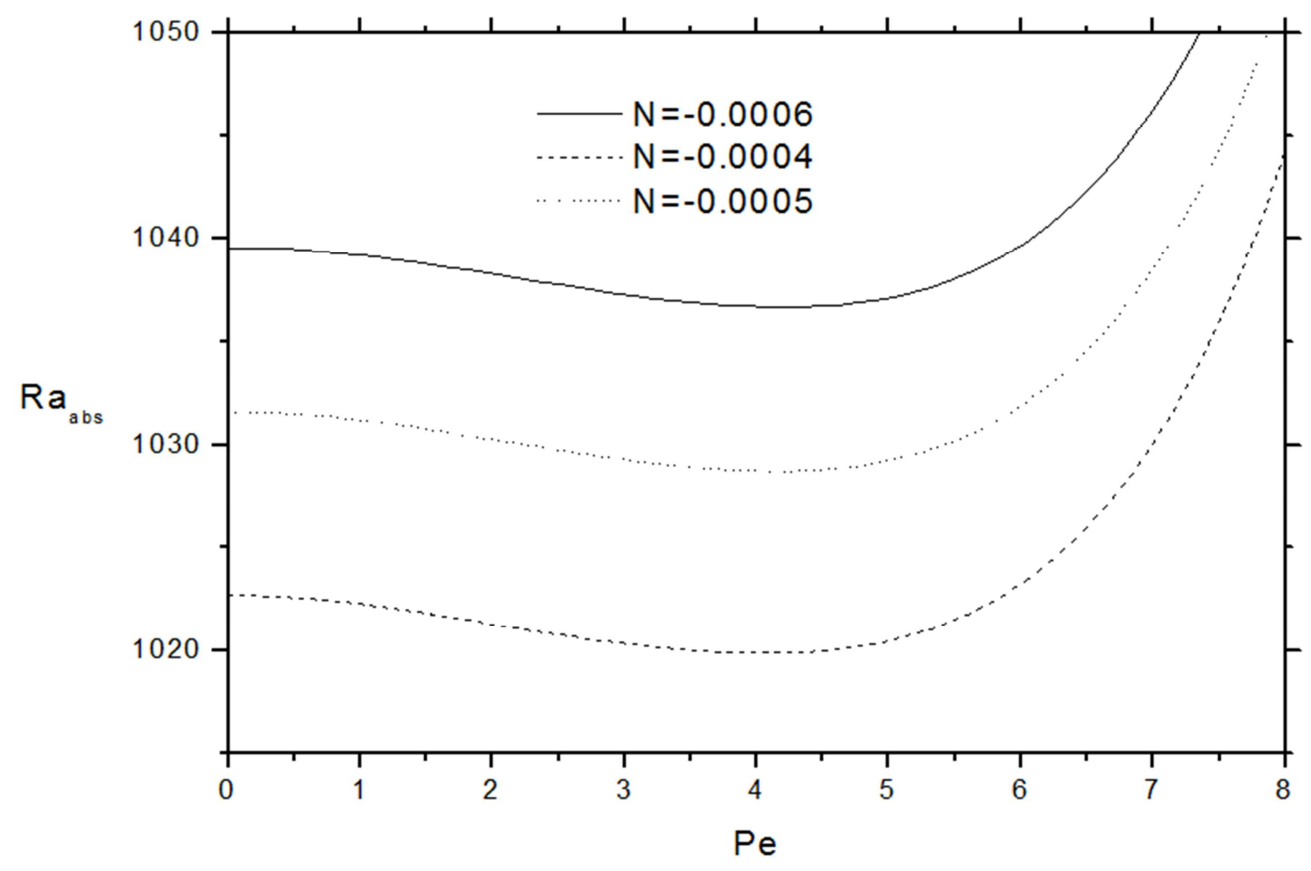

Figure 6. Variation of Ra with Pe for negative $N, L e=140$.

Solving the perturbation equations by a variational method, shows the physical meaning of a complex wave number $k_{x}$ accompanying the absolute instabilities, it corresponds to a wave amplitude varying according to the $\mathrm{x}$-coordinate. For the case of a semi-infinite cavity, a thermal area settles at the entry of the canal. Figure 7 is the graphical representation of the eigenvector of the absolute threshold. Through this representation one sees the growth of the amplitude of perturbations (bottom) and global flow (top). The different simulations shows that the length of the area of establishment depends on the absolute threshold, and essentially on the $P e$ number.

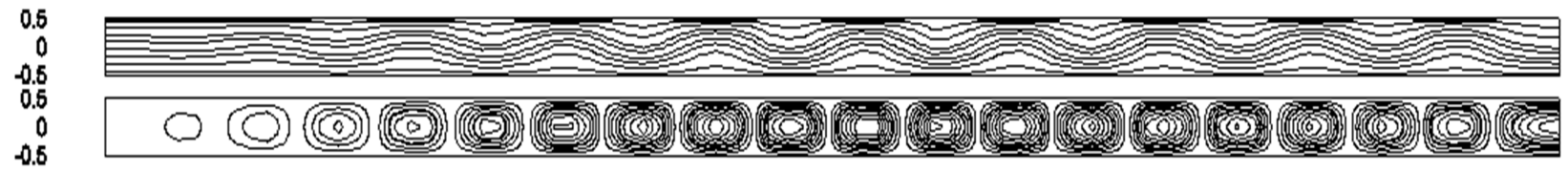

Figure 7. Spatial amplitude growth and thermal inlet area; $P e=1, R a_{a b s}=1039, N=-0.0006$.

\section{Conclusion}

In the present work, we have studied the stability of binary liquid layer sliding in motion between two other fluids. The problem is theoretically interesting because it allows an analytical solution.

Generally, in the absence of throughflow the system has a stationary property at the threshold of convection for positive $\mathrm{N}$ and oscillatory properties for negative $\mathrm{N}$. In both cases, none spatial direction is privileged. However; in presence of throughflow the system loses its stability via a frequency shift. For convective instabilities, the problem has analytical solution and is a Galilean transformation of natural convection. The advection due to throughflow makes the rolls to propagate and breaks Galilean invariance at the absolute instabilities threshold. Then, the symmetry by the rotational invariance is broken, and the system selects among an infinite number of unstable modes, the progressive waves, in the direction of the flow. The absolute Rayleigh numbers are given according to $\mathrm{Pe}$ and $\mathrm{N}$ numbers.

\section{Nomenclature}

$x, z$ : Coordinate system

$L_{x}, L_{z}$ : Length and thickness of the layer

$u, w$ : The velocity components

$A_{x}:$ Aspect ratio $A_{x}=L x / L z$

$T:$ Temperature

$C$ : Mass fraction

$D_{C}$ : Mass diffusivity $\left[\mathrm{m}^{2} \mathrm{~s}^{-1}\right]$

$D_{T}$ : Thermodiffusion coefficient $\left[m^{2}(s K)^{-1}\right]$

$g$ : Gravity acceleration $\left[\mathrm{m} / \mathrm{s}^{2}\right]$

$L e$ : Lewis number $L e=\kappa / D_{C}$

$P r$ : Prandtl number $\operatorname{Pr}=v / \kappa$

$N$ : Buoyancy ratio $N=S_{T} \times\left(\frac{\beta_{\phi}}{\beta_{T}}\right) C_{0}\left(1-C_{0}\right)$

$R a$ : Rayleigh number $R a=\frac{g \beta_{T} \Delta T L_{z}{ }^{3}}{(v \kappa)}$

$R e$ : Reynolds number $R e=\frac{u L_{z}}{v}$

$P e$ : Peclet number $P e=R e \times P r$

$k$ : X-direction wavenumber 
$S_{T}:$ Soret coefficient $S_{T}=\frac{D_{T}}{D_{C}}$

$\mathcal{R}^{0}:$ Natural convection constant $\frac{27 \pi^{4}}{4}$

\section{Greek Symbols}

$\beta$ : Volumetric expansion

$\kappa$ : Thermal diffusivity $\left[\mathrm{m}^{2} \mathrm{~s}^{-1}\right]$

$v$ : Kinematic viscosity $\left[\mathrm{m}^{2} \mathrm{~s}^{-1}\right]$

$\omega$ : Adimensioned pulsation

$\theta$ : Temperature perturbation

$\phi$ : Mass fraction perturbation

\section{References}

[1] D.A. Nield, C.T. Simmons, A.V. Kuznetsov, and J.D. Ward, On the Evolution of Salt Lakes: Episodic Convection beneath an Evaporating Salt Lake, Water Resources Research, Vol. 44, article W02439, pp. 1-13, 2008

[2] J. Hu, H. Ben Hadid, and D. Henry, Linear stability analysis of Poiseuille-Rayleigh-Bénard flows in binary fluids with Soret effect, Phys. Fluids 19, 034101 (2007)
[3] J.K. Platten, J.C. Legros, Convection in Liquids, SpringerVerlag, Berlin, 1984

[4] H.W. Müller, M. Lücke, Transversal convection patterns in horizontal shear flow, Physical Review A, Vol. 45, Number 6, pp. 3714-3726, 1992

[5] Ben Hamed H., Bennacer R., Sammouda H., PoiseuilleRayleigh-Bénartd problem in a horizontal duct of finite length in streamwise direction: stability analysis, Progress in Computational Fluid Dynamics, Vol. 8, Nº6, pp. 342-350, 2008

[6] Carrière P., Monkewitz P.A., Convective versus absolute instability in mixed Rayleigh-Bénard-Poiseuille convection, Journal of Fluid Mechannics, Vol 384, pp. 2198-2207, 1999

[7] A Joulin. M.N Ouarzazi., Mixed convection of a binary mixture in a porous medium, C.R Acad. Sci. Paris, t, 328, Série II b, pp. 311-316, 2000

[8] T. A Campbell, J. N Koster.: Modeling of liquid encapsulated Gallium melt. Acta Astron. Vol. 35, pp. 805-812, 1995.

[9] S Chandrasekhar, Hydrodynamic and Hydromagnetic Stability, Oxford University, 1961 